\title{
Piezoelectric Polymer Actuator and Material Properties
}

\author{
V.H. Schmidt, D. Brandt, F. Holloway', A. Vinogradov', and D. Rosenberg ${ }^{1}$ \\ Physics and 'Mechanical Engineering Departments \\ Montana Stale University, Bozeman, MT 59717
}

\begin{abstract}
This paper presents the construction and performance of a $\mathrm{PVF}_{2}$ (poly(vinylidene fluoride), $\left(\mathrm{CH}_{2} \mathrm{CF}_{2}\right)_{\mathrm{l}}$ thin film piezoelectric actuator. In addition, the paper discusses the methods used to characterize the viscoleastic properties of the actuator material.
\end{abstract}

\section{INTRODUCTION}

Development of a low force, large displacement actuator constructed of $\mathrm{PVF}_{2}$ is currently underway for aclive vibration sufpression of instrument packages in orbiting spacecraft. Because $P F_{2}$ is a low cost, lightrieight, robust, piezoelectric material that can easily be shaped for specific applications, it is ideally suited for this type of actuator. Several actuator designs have been constructed and evaluated under a variety of conditions. Research is also currently in progress conceraing the mechanical testing and material characterization of $\mathrm{PVF}_{2}$. Static tests indicate that the mechanical behavior of $\mathrm{PVF}_{2}$ thin films is time dependent, i.e. samples of this material creep under room lemperature conditions. Because socurate predictions of the timedependeat effects due to the viscoelastic properties are critical in the design of active vibration control devices, material characterization of $\mathrm{PVF}_{2}$ is also in progress.

\section{Actuator Design ALD CONSTRUCTION}

The primary component of the current actuator design, Fig. 1, is a thin sheet of $\mathrm{PVF}_{2}$ film, 28 microns thick, about $3.4 \mathrm{~cm}$ wide by $5 \mathrm{~cm}$ long and electroded on both sides. The sheets are currently purchased prefabricated and electroded. The electrode on one side covers the entire sheet while the opposite side has narrow electrode gaps at $1 / 4$ and $3 / 4$ of the distance along the stretch or longitudinal (1) direction, see Fig. 2. Each balf of the actuator itself is made up of a bimorph design incorporating two sheets of oppositely poled pobmer film glued together with a conductive epoxy. The thickness of the glue layer is, on average, 30 microns. The bimorph is formed into a double-S curve during manufacture. Applying a field to the middle and an opposite field at the ends of the central bimorph electrodes will accentuate or diminish the curvature depending upon the field polarity. The degree of curvature is carefully chosen such that when the actuator is activated there nill be enough space between the bimorphs so that the maximum applied field will not be enough to bring the two bimorphs together. In this way, the displacement will be close to a limear finction of applied voltage. For the final stage of

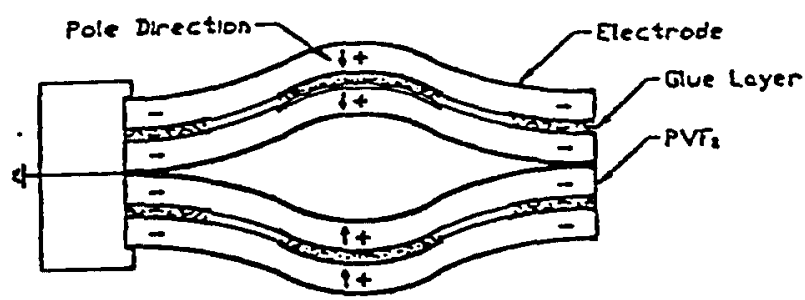

Figure 1: $\mathrm{PVF}_{2}$ Actuator Design.

construction two of the bimorphs are glued together at the ends nith a conductive epoxy to complete the actuator.

\section{THEORY OF OPERATION}

The particular film utilized in this work has a $d_{31}$ boundary condition, see Fig. 2. In short, the film is free to move in the transverse (2) and thiclness (3) directions while an applied electric field across the (3) direction will result in a change in length in the longitudinal (1) direction [1].

The distortion of the $\mathrm{PVF}_{2}$ film (shonn in Fig. 3) as a result of an applied electric field is defined by the relation:

$$
S_{1}=d_{31} E
$$

where $S_{1}=\Delta L /$ and represents the strain in the longitudinal direction at a certain field strength $E(V / h)$ and $d_{31}$ is the piezoelectric strain constant. Rearranging the equation gives the formula:

$$
\Delta L=d_{31}(V / t) L
$$

and shows that the change in length depends only upon the applied voltage because the length and thickness remain essentially constant. This condition of cbanging length is taken sdvantage of in the bimorph design. As shomn in Fig. 4, the applied electric field will cause one film to elongate while the other contracts, resulting in an overall bending motion upward or downward. 


\section{ACTUATOR RESPONSE}

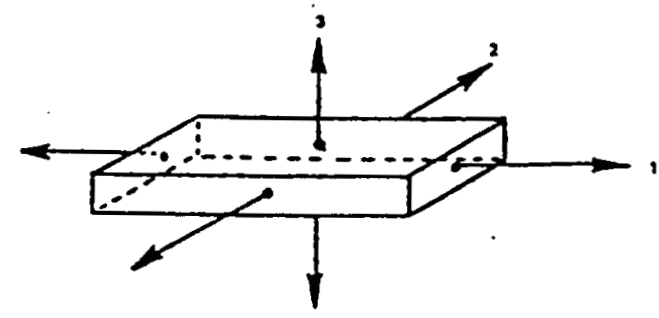

Once assembled these actualors were tested for their response to various voltages at different frequencies. A test mechanism was designed to measure the peak to peak displacements of the actuators, see Fig. 5. This device consisted of using a mirror attached to a pivot which is then rotated by a lever arm that follows the motion of the actuator. A laser beam is reflected by a mirror onto a screen where the maximum travel of the beam is recorded. The peak to peak travel of the actuator $(\Delta x)$ is related to the mirror's angle of rotation $(\phi)$ by the relation:

$$
\left.\Delta x=\sqrt{\left(2 l^{2}\right.}(1-\cos \phi)\right)
$$

Fig. 2. Stress/Strain Coordinate System



where $\phi$ is calculated by:

$$
\phi=\frac{\operatorname{atan} \frac{H_{f}}{L}-\operatorname{atan} \frac{H_{0}}{L}}{2}
$$

A waveform generator was used to provide an alternating current signal of various low frequencies. This signal was split and sent through an inverting circuit with a gain of 1 . This provided two signals, one $180^{\circ}$ out of phase with the other. Both

Fig. 3. Relative Deformation of Film

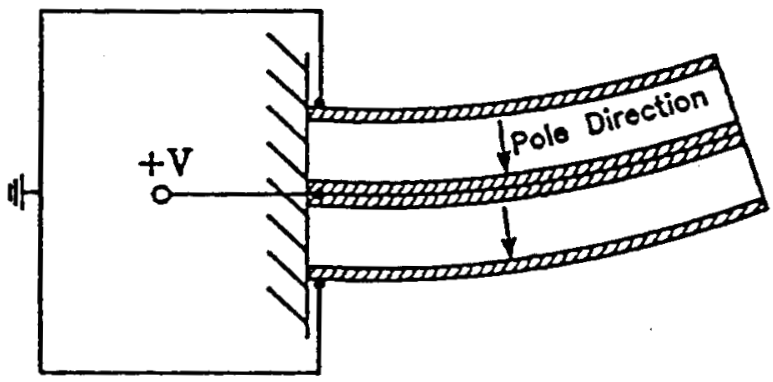

Fig. 4. Electro-Mechanical Actuation of Bimorph

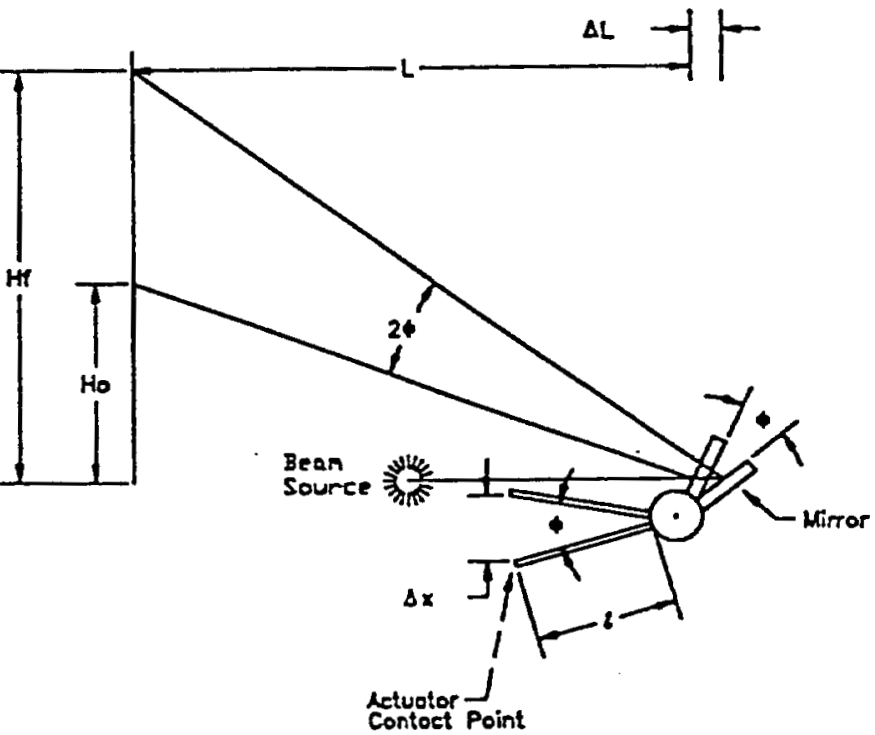

Fig 5: Technique for Actuator Measurement. Note: $\Delta \mathrm{L}$ is very small compared to $\mathrm{L}$ and was thus ignored in the displacement calculations. 
signals were sent through an amplifier and then to the innermost electrodes of the test actuator, leaving the outside electrodes to be connected to ground. The applied voltages were measured at the actuator with an oscilloscope and recorded as peak to peak voltages. The test voltages were limited by the recommended maximum operating voltage of $30 \mathrm{~V} / \mu \mathrm{m}$ [1] but experience showed we were able to go as high as $36 \mathrm{~V} / \mu \mathrm{m}$ without any adverse affects on the actuator. Fig. 6 shows the results of these tests for a typical $\mathrm{PVF}_{2}$ actuator.

The actuator deflections nere fairly symmetrical about their original or relaxed positions. In addition, the devices showed mechanical resonance peaks. As voltages were increased across the actuators, the frequencies at which these peaks occurred would decrease. The exact peak frequencies were unique for each actuator tested but each showed the same behavior pattern. It was also observed that the DC end of the spectrum $(0 \mathrm{~Hz})$ exhibited larger displacements than the AC portion from $1-10 \mathrm{~Hz}$. This behavior was attributed to the relaxation of the glue layer during the DC portion of the tests.

The maximum recorded displacement was $3.89 \mathrm{~mm}$ peak to peak at a field of $36 \mathrm{~V} / \mu \mathrm{m}$ and frequency of $13 \mathrm{~Hz}$. In addition, certain displacements (notably those voltages above $500 \mathrm{~V}$ p-p at frequencies above $10 \mathrm{~Hz}$ ) were not able to be recorded due to the devices' tendency to accelerate beyond $\mathrm{lg}$ and thus "bounce" the lever arm, making measurement impossible. The large displacements in the low frequency ranges make these devices ideally suited for the anticipated vibration frequencies aboard orbiting spacecraft.

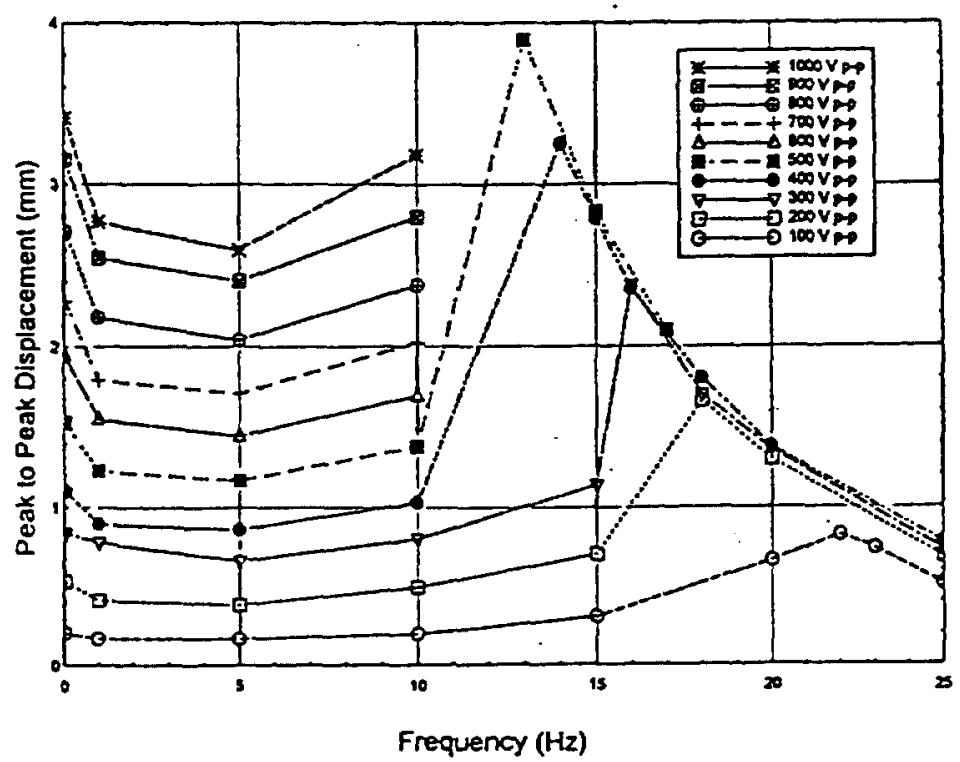

Fig. 6. Actuator Displacement Vs. Frequency

\section{Materlal Characterization}

A review of current approaches to modeling the material response of piezoelectric thin film polymers indicate that, at present, little or no work has been done to take into account the time-dependent behavior of these materials. Typically, such materials are treated as linearty elastic, and their respective constitutive model is obtained by coupling the linear elastic law with piezoelectric relations [2]. A research program with the objectives to characterize the viscoelastic properties of $\mathrm{PVF}_{2}$ thin films and to formulate a constitutive model incorporating the time dependent behavior of these materials has been developed. This work includes dynamic testing of the thin film under room and elevated temperature conditions.

The dynamic experimental program was developed using the theoretical approach outlined in Ref. [4] and applied in Ref. [5]. The material properties are primarily defined by the complex moduli and the loss factor, which are functions of frequency, temperature, strain amplitude and prestress. Static creep tests could be used to determine the creep compliance, however, these tests require long periods of time. Dynamic tests allow measurements of the complex moduli in the frequency domain, which allows very rapid characterization, and eliminates the need for tests in the time domain [3].

The first step in evaluating material properties in a dynamic test is to determine the complex compliance in the frequency domain by measuring phase shift and ratio of peak stress to peak strain. A mechanical testing devise is used to provide a periodically oscillating stress. $28 \mu \mathrm{PVF}_{2}$ samples were prestressed to maintain a tensile load during the testing. The resulting strain also oscillates with the same period, but is out of phase with the stress. From [3], the storage modulus and the loss modulus can be calculated as:

$$
E^{\prime}(\omega)=\frac{\sigma^{\circ}}{\varepsilon^{\circ}} \cos \delta, \quad E^{\prime \prime}(\omega)=\frac{\sigma^{\circ}}{\varepsilon^{\circ}} \sin \delta,
$$

where $\delta$ is the phase angle between peak stress $\left(0^{\circ}\right)$ and peakstrain $\left(\epsilon^{c}\right)$. E' $(\omega)$ is the extension storage modulus and is directly proportional to the average energy storage in a cycle and E' $(\omega)$ is the extension loss modulus and is proportional to the average loss of energy as heat in a cycle.

The complex modulus is then given as :

$$
E^{\cdot}(\omega)=E^{\prime}(\omega)+i E(\omega)^{\prime \prime}
$$

This immediately yields the complex compliance since: 


$$
\begin{gathered}
D^{\cdot}(\omega)=\frac{1}{E^{\cdot}(\omega)}=D^{\prime}-i D^{\prime \prime} \\
D^{\prime}(\omega)=\frac{1}{E^{\prime}(\omega)\left(1+\tan ^{2} \delta\right)}=\frac{\varepsilon^{0}}{\sigma^{\circ}} \cos \delta \\
D^{\prime \prime}(\omega)=\frac{1}{E^{\prime \prime}(\omega)\left(1+\left(\tan ^{2} \delta\right)^{-1}\right)}=\frac{\varepsilon^{\circ}}{\sigma^{\circ}} \sin \delta
\end{gathered}
$$

By measuring the phase angle and the ratio of maximum amplitude of stress to strain over a streep of frequencies at various temperatures and from (8) and (9), graphs such as Fig. 7 are produced for each prestress and temperature.



Figure 7. Storage and Loss Modulus for Thin Film $\mathrm{PVF}_{2}$ at Constant Prestress (23.56\% Yield) and Temperature $\left(21^{\circ} \mathrm{C}\right)$.

The second step uses theories of time-lemperature and stresstime correspondence to develop a master cun'e which describes the moduli or loss factor over a wider range of frequencies than was measured An increase in temperature is nearly equivalent to a decrease in frequency, if linear viscoelastic behavior exists. A temperature range of $100^{\circ} \mathrm{F}$ may provide an effective frequency of 10 logarithmic decades. Producing the reduced variable plots is done by first choosing a reference temperature, then determining empirically the horizontal shift required to superpose the curves. These values are recorded as $\Delta \log \mathrm{a}_{\mathrm{T}}$. The values of $\Delta \log$ a $_{r}$ are then added progressively from $T_{0}$ to obtain $\log$ a at each temperature. Since not all materials exhibit the temperaturefrequency correspondence, it is vital that the curves be able to superpose nearly exactly and that the same values of $a_{T}$ be able to superpose all viscoelastic functions for the method to be valid.

The original recorded viscoel astic function (shifted vertically by $\left.T \rho / T_{0} p_{d}\right)$ is then plotted against $\log \omega_{2}$ to obtain the master shif curve. From these curves, the viscoelastic function can be obtained at any other desired temperature by reversing the reduction.

A unified master curve that incorporates both $\mathrm{a}_{\mathrm{J}}$ and $\mathrm{a}_{\mathrm{a}}$, a stress dependent shifting factor found in a manner similar to $a_{n}$ is made by plotting the viscoelastic function (shifted vertically by $\left.T \rho / T_{2}\right)$ vs $\log \left(\omega a_{a_{0}} a_{0}\right.$. The method of Ninomiya and Ferry [2] is then used to transform the loss and storage compliance obtained with dynamic-mechanical tests to the creep compliance in the time domain:

$$
D(t)=D^{\prime}(\omega)+0.40 D^{\prime \prime}(0.4 \omega)-0.014 D^{\prime \prime}(10 \omega) \quad t=\frac{1}{\omega}
$$

Thus, the crep compliance is bnorn and can be used to predict the time dependent response of the actuator.

During the dyamic testing, the voltage produced by the piezoelectric sample is also recorded. This will establish whether the piezoelectric coefficient is dependent on temperature and prestress. Long time oscillatory tests nill determine if the piezoelectric properties vary significantly with time. Efforts nill then be made to develop a constitutive model for combined viscoelastic-piezoelectric properties.

\section{REFEREICES}

(1) KINAR Pieso Film Technical Mfonual, Ponwath Corporation, 1983, pp. 30-34, 37-39, 51.

[2] Takuro Dkods, Fundamenials of Picoelectricity, Oxford University Press, 1990, p. 263

[3] J. D. Fenr, Viscoelastic Properties of Polymers, 3rd ad, Jobn Wiley \&e Sons, 1980, pp. 11-30,90, 264280.

[4] Schapery, R.A, "On the Coradarization of Nonliocar Viscoelaste Mrtcrials", Polymer En gioering and Science, No. 9, 1969, p. 295.

[5] H. Golden, T. Straganac, and R Schapery, "A Tes and Aoelysis Prodocol to Rapidly Charadaize Nonlinear liscoelasjic Properties of Balloon Mirtorials", AlAA 96-0572, 34h Aoropace Sciences Meaing and Exhibit, Jan. 15-18, 1996, Reno, NV. 